\title{
Football Result Prediction Based on Machine Learning
}

\author{
Wenbo $\mathrm{YU}^{1}$ \\ Faculty of Science and Technology, Beijing Normal University-Hong Kong Baptist \\ University United International College, China
}

\begin{abstract}
By constructing features such as goal difference, FIFA team ratings and league points, and by using machine learning technologies such as logistic regression and support vector machine, appropriate models are constructed to make binary prediction of match results.
\end{abstract}

Keywords. Logistic regression, support vector machine, predictive model, machine learning.

\section{Background Introduction}

In recent years, more and more people pay attention to statistics, and football is no exception. Although football data analysis is still a developing field, with the rise of the information age in the 21 st century, data is playing an increasingly important role in football. For example, there are academics who use increasingly sophisticated machine learning techniques to predict the outcome of games. In this project, objective data analysis is mainly used to predict the results of premier League matches by using logistic regression, SVM and other models, and also evaluate the performance of the model and predictive results. (The Premier League is one of the top five leagues in European football, with 380 games played each season.)

\section{Literature Review}

As a popular sport, football has been studied by many scholars. For example, Cao Weimin and Shi Zhishe [1] analyzed the 2000 European Football Championship finals by using the methods of literature, observation, statistics and comparative research. Hou Huisheng, Zang Hepeng, Li Fengqiao [2] used Q-type clustering, rank correlation analysis and multiple comparative statistical methods to analyze the index data of 11 attacks in 64 matches of the 2006 World Cup Finals. In addition, in terms of football data modeling, Karlis and Ntzoufras[3] also used binary Poisson distribution model to illustrate football data. Fenton and Neil[4] proposed the Pi-Football model based on Bayesian network to predict the results of Football matches. The research results of

1 Corresponding Author, Wenbo YU, Faculty of Science and Technology, Beijing Normal University-Hong Kong Baptist University United International College, China; E-mail: 1628463139@qq.com. 
Gudmundsson \& Wolle[5] are more commercialized. They developed a series of automatic football analysis tools, together constitute a complete football data analysis software.

\section{Methodology}

\subsection{Dataset Description}

Two football datasets were used for analysis. The first data sets the FIFA game data set (https://sofifa.com/players?r=210006\&set=true\&col=oa\&sort=desc) in this website, we can find related to the players, the team and league FIFA game data, Examples include individual player ratings, numerical values, and overall offensive and defensive team ratings.

The second dataset used data from UK football betting websites. (http://football-Data.co.uk/englandm.php) On the second website, we can find football data related to different leagues, which can effectively help us get reliable league football data in the first time.

Data tables are provided on the site, which contain a range of attributes such as final match results, home and away teams, goals scored, shots times, and can be used to create new features to improve the performance of the model.

\subsection{Application Method}

Firstly, we will prepare the fitting data by creating available features through four steps of data selection, data cleaning, feature engineering and heat map, and use the logistic regression, SVM and XGBoost model provided by SKlearn to fit the data of premier League season 16-19 to achieve the prediction of match results. Finally, the F1 score and accuracy evaluation model are used.

\subsubsection{Data Selection}

Before creating the right model, we need to select the right data set to fit the model. We tried all data sets from 2000 to 2019 , and determined that the selected year data should not be too far away from the predicted season, and the selected season data should not be too few, otherwise the model may not fit well and the generalization ability is not strong enough to have good prediction. After comparing the performance of the model with data sets of different seasons, we finally decided to use 16-19 seasons as the input data set of the model.

\subsubsection{Data Cleaning}

Missing data was found in the 2018 season dataset as one of the inputs and the data for this season was deleted.

\subsubsection{Feature Engineering}

(Note: Data from the first three weeks of each season was removed from the experiment, due to a lack of historical information to predict at the beginning of the season.) 
Table 1. Feature created

\begin{tabular}{|c|c|}
\hline Feature & Description \\
\hline Goal difference for home and away teams H/ATGD & Goals scored - Goals conceded \\
\hline Points for home and away teams H/ATP & Win $=3$, draw $=1$, lose $=0$ \\
\hline Each team's last three game performances H/AM & $\begin{array}{c}\text { Home team: } \text { win }=\text { win; draw or lose }=\text { lose } \\
\text { Away team: win or draw }=\text { win; lose }=\text { lose }\end{array}$ \\
\hline FIFA ratings for home and away teams H/AR & FIFA game ratings \\
\hline
\end{tabular}

From the table 1, four features are created and a matchweek feature is added to the data sheet, i.e. the matchweek in which the match took place. Goal difference and team points can be averaged to better fit the model. After creating the features, we looked at wins and losses in the Premier League and we found that from the table 2 the home team had a winning rate of $47.43 \%$, proving that the Premier League does have a home advantage. As a result, the tag ratio is unbalanced on win, lose and draw issues. Therefore, we use a binary classification problem of whether the home team will win or not, which is also a way to solve the problem of unbalanced tag ratio.

Table 2. Home court situation

\begin{tabular}{|c|c|}
\hline \multicolumn{2}{|c|}{$16-\mathbf{1 9}$ seasons PL data } \\
\hline Total matches & 1050 \\
\hline Total features & 12 \\
\hline Home team win & 498 \\
\hline Home team win rate & $47.43 \%$ \\
\hline
\end{tabular}

3.2.4. Heat Map

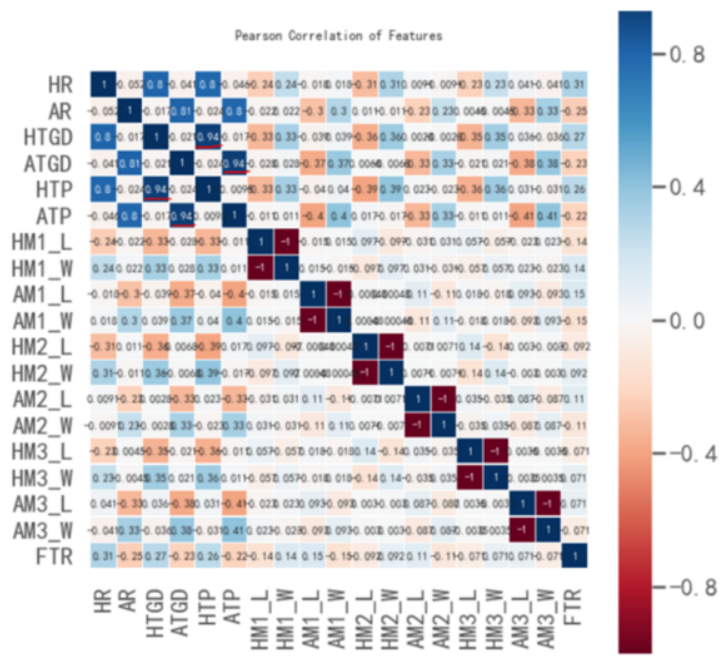

Figure 1. Heat map 
The figure 1 above shows a strong correlation between HTP and HTGD, as well as between ATP and ATGD. The higher the average score, the higher the goal difference. In order to avoid multicollinearity, the H/ATP feature of league points was deleted while the H/ATGD feature of goal difference was retained. However, given that multicollinearity only appeared in logistic regression models, the league point H/ATP feature was continued to be used in other models to predict results.

\subsection{Model Building}

Table 3. Model building and parameters

\begin{tabular}{|c|c|}
\hline API Interface & Parameters \\
\hline Sklearn - Train_test_split & X_all, y_all, test_size $=0.3$ \\
\hline Sklearn - LogisticRegression & $\begin{array}{c}\text { Solver = 'liblinear' } \\
\text { class_weight = 'balanced' }\end{array}$ \\
\hline Sklearn - SVC & $\begin{aligned} \text { Kernel } & =\text { ' rbf } \\
\text { class_weight } & =\text { ' balanced }\end{aligned}$ \\
\hline XGBoost & booster = 'gblinear' \\
\hline Sklearn - fl_score & target, y_pred, pos_label $=1$ \\
\hline Accuracy & 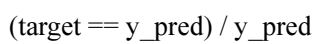 \\
\hline
\end{tabular}

From the table 3, we have built the models and evaluation methods, then tried to tune the XGBoost.

\begin{tabular}{|c|c|}
\hline & Using GridSearchCV to adjust parameters \\
Sklearn - GridSearchCV & 'n_estimators': $[80,90,100,110,120]$ \\
& 'max_depth': $[3,4,5,6,7,8,9]$ \\
\hline
\end{tabular}

\section{Experimental Results and Analysis}

Table 4. Performances

\begin{tabular}{|c|c|c|c|c|c|}
\hline Model & $\begin{array}{c}\text { F1 scores in } \\
\text { training set }\end{array}$ & $\begin{array}{c}\text { Accuracy of } \\
\text { training set }\end{array}$ & $\begin{array}{c}\text { F1 scores in } \\
\text { test set }\end{array}$ & $\begin{array}{c}\text { Accuracy of } \\
\text { test set }\end{array}$ & $\begin{array}{c}\text { Sample } \\
\text { size }\end{array}$ \\
\hline $\begin{array}{c}\text { Logistic } \\
\text { regression }\end{array}$ & 0.6573 & 0.6680 & 0.6601 & 0.6730 & \\
\cline { 1 - 4 } SVM & 0.7041 & 0.7129 & 0.6465 & 0.6667 & \multirow{2}{*}{735} \\
\cline { 1 - 4 } XGBboost & 0.4889 & 0.5310 & 0.5310 & 0.6635 & \\
\hline Enhanced XGB & 0.4861 & 0.6231 & 0.5244 & 0.6603 & \\
\hline
\end{tabular}

It can be seen from the table 4 that in the training set, SVM had the best performance in F1 score and accuracy, reaching about $70 \%$, while in the test set, logistic regression had a better overall performance, maintaining an overall accuracy of about $67 \%$. The accuracy of other models was also good, reaching about $66 \%$. However, the results so far are only binary predictions in an idealized state. It is very difficult to predict the real results of a football match, there is no prediction model or institution that can achieve a predicted winning rate of more than $60 \%$. Even the odds on betting websites tend to 
come up with something unexpected, with so many random factors influencing the game that weak teams tend to pull off unexpected reversals.

The next four prediction models are used to briefly predict some premier League matches for the 2020-2021 season. (away)

Match prediction 1: 2020/11/22 Tottenham Hotspur (home) VS Manchester City

Table 5. Predictive result 1

\begin{tabular}{|c|c|}
\hline Model & Predictive results \\
\hline Logistic regression & Home didn't win \\
\hline SVM & Home win \\
\hline XGBoost & Home win \\
\hline Tuning XGBoost & Home win \\
\hline
\end{tabular}

Final result: Tottenham hotspur 2-0 win for Manchester City, table 5 shows 3 models giving the correct answer.

Match prediction 2: 2020/11/23 Liverpool (home) VS Leicester City (away)

Table 6. Predictive result 2

\begin{tabular}{|c|c|}
\hline Model & Predictive result \\
\hline Logistic regression & Home win \\
\hline SVM & Home win \\
\hline XGBoost & Home didn't win \\
\hline Tuning XGBoost & Home didn't win \\
\hline
\end{tabular}

Final result: Liverpool 3-0 to Leicester city, table 6 shows the 2 models giving the correct answer.

\section{Conclusion}

By using various objective data of football matches to create suitable features to fit more suitable models, it has been possible to achieve rough prediction of football match results. However, these models still remain in binary prediction, that is, the only prediction result is whether the home team win or not, and the prediction accuracy is about $60 \%$. This probability is still quite different from the actual probability, so more available features and models need to be considered to improve the range and accuracy of prediction, and provide reference for subsequent research on football prediction.

\section{References}

[1] Shi Zhshe, \& Cao Weimin, (2002). On the Relationship between shooting position and goal -- Statistical Analysis of shooting data in European Football Championship 2000. Hubei Sports Science and Technology, 021(002), 228-230.

[2] Hou Huisheng, Zang Hepeng, \& Li Fengqiao. (2008). Comprehensive Evaluation of the Offensive Ability of soccer teams in the 2006 World Cup. Journal of Beijing Sport University, 031(001), 138-140,143.

[3] Karlis, D., \& Ntzoufras, I. (2003). Analysis of sports data by using bivariate Poisson models. Journal of the Royal Statistical Society: Series D (The Statistician), 52(3), 381-393.

[4] Constantinou, A. C., Fenton, N. E., \& Neil, M. (2012). pi-football: A Bayesian network model for forecasting Association Football match outcomes. Knowledge-Based Systems, 36, 322-339.

[5] Gudmundsson, J., \& Wolle, T. (2014). Football analysis by using spatio-temporal tools. Computers, Environment and Urban Systems, 47, 16-27. 